\title{
AVALIAÇÃO DAS TÉCNICAS DE HEMOAGLUTINAÇÃO INDIRETA E IMUNOFLUORESCÊNCIA INDIRETA NO DIAGNÓSTICO DA AMEBÍASE INTESTINAL
}

\author{
Edward Félix Silva , Maria Aparecida Gomes", Maria das Graças Carvalho **
}

\section{RESUMO}

Testes de hemoaglutinação indireta (HAI) e imunofluorescência indireta (IFI), foram realizados em 154 soros de indivíduos de ambos os sexos comprovadamente infectados com Entamoeba histolytica. Dentre estes 154 soros, 78 foram obtidos de pacientes com sintomas clínicos de amebíase (colite disentérica e colite não disentérica) e os restantes 76 soros de indivíduos assintomáticos. Os antigenos foram preparados das cepas HK-9, NIH-200 e ICB-462, todas mantidas em cultivo axênico. Os resultados obtidos, tanto para HAI como para IFI, foram analisados e relacionados à forma clínica, idade e sexo do paciente. Foi encontrada maior positividade nas reaçóes de pacientes com idade superior a 18 anos (IFI: 12/21; HAI: 10/23) e apresentando sintomas clínicos (IFI: 21/57; HAI: 16/62).

UNITERMOS: Amebíase; Diagnóstico; Imunofluorescência indireta; Hemoaglutinação indireta.

\section{INTRODUÇÃ̄O}

Estima-se que 500 milhð̌es de pessoas, no mundo, se encontrem infectadas pelo protozoário parasito Entamoeba histolytica. Dentre estes indivíduos infectados, uma percentagem relativamente pequena desenvolve sintomas da doença. A ameblase sintomática é grave e muitas vezes fatal, levando a morte anualmente entre 40 a 110 mil pessoas (GUERRANT, 1986). O fato de um grande número de indivíduos com amebíase não apresentarem sintomas e dos indivíduos sintomáticos estarem restritos a determinadas áreas do globo, levou diversos pesquisadores nesta área a concordarem com a hipótese de Brumpt (1925), que sugere a dualidade de espécies, sendo a $E$.

- Depto. Parasitologia, Instituto de Ciências Biológicas-UFMG, CP 486, CEP: 31270-901.

* Depto. Análises Clínicas e Toxocológicas da Faculdade de Farmácia da Universidade Federal de Minas Gerais (UFMG).

Recebido 08/04/96. Revisto em 26/08/96. Aceito em 05/09/96. 
SILVA, E.F.; GOMES, M.A.; CARVALHO, M.G.Avaliação das técnicas de Hemoaglutinação Indireta e Imunofluorescência Indireta no diagnóstico da amebíase intestinal. Rev. Pat. Trop.25 ( 1 ): 5-11, jan/jun. 1996

dispar, morfologicamente idêntica a E. histolytica, a forma infectante presente nos indivíduos assintomáticos. Considerando esta nova situação, indivíduos infectados com $E$. dispar seriam dispensáveis de tratamento e sem significância epidemiológica, fazendo-se necessário repensarmos a distribuição da $E$. histolytica e da $E$. dispar no mundo. Para estudos epidemiológicos, os testes sorológicos são os mais indicados por serem simples, rápidos, não precisarem, na maioria dos casos, de microscopistas treinados. Dentre as diversas provas sorológicas que foram testadas para detectar anticorpos e antígenos amebianos circulantes (GANDHI et al., 1987; NEAL et al., 1968), a hemoaglutinação indireta e a imunofluorescência indireta, são sensíveis, específicas e amplamente utilizadas no diagnóstico da amebíase e em levantamentos epidemiológicos (HEALY, 1986; KESSEL et al., 1965; KNOBLOCH et al., 1982; NEAL et al., 1968; SAVANAT et al., 1974; STAMM et al., 1976). A imunofluorescência indireta tem sido também usada como prova de cura em amebíase (CUNHA et al., 1977; STAMM et al., 1976).

Neste trabalho, foram utilizados soros de indivíduos infectados com $E$. histolytica apresentando diferentes quadros clínicos (colite disentérica [CD], colite não disentérica [CND] e assintomáticos) analisando-se a efetividade destas técnicas no imunodiagnóstico da amebíase no Brasil, onde predominam assintomáticos e sintomáticos com CND.

\section{MATERIAL E MÉTODOS}

\section{Soros humanos:}

Para escolha dos soros utilizados neste estudo, foi feita uma pré-seleção dos indivíduos através de exame de fezes pelos métodos de conservação das fezes com mertiolate, iodo e formol, formol-éter e exame direto e coloração pela hematoxilina férrica nos casos de fezes diarréicas. Foram considerados os indivíduos que apresentaram em suas fezes cistos ou trofozoítos de $E$. histolytica. Foram encontrados positivos 154 indivíduos de ambos os sexos, com idade entre 6 e 60 anos. Foi feito o exame clínico destes indivíduos e colhido o sangue para obtenção do soro. Dos 154 positivos, 78 apresentaram sintomas de infecção, sendo 2 com CD e 76 com CND. Os restantes 76 eram assintomáticos. Utilizamos soros controles positivos e negativos da Ames Company - (Division Miles Laboratories Inc., Indiana, USA) e soros negativos obtidos de recém-nascidos, filhos de mães isentas de infecção pela E. histolytica. Foram utilizados ainda como controles 5 soros de indivíduos infectados unicamente com E. coli, 2 com E. coli e E. hartmanni, 1 com Iodamoeba butschlii e Endolimax nana e 1 com E. coli e Dientamoeba fragilis.
SILVA, E.F.; GOMES, M.A.; CARVALHO, M.G.Avaliaçăo das técnicas de Hemoaglutinaçăo Indireta e Imunofluorescência Indireta no diagnóstico da amebíase intestinal. Rev. Pat. Trop.25 ( 1 ): 5-11, jan/jun. 1996

\section{Antigenos:}

Foram utilizadas as cepas HK-9 (considerada como cepa padrão [KNOBLOCH et al., 1982; GOLDMAN, 1966; SAVANAT et al., 1974]) e a cepa NIH-200, isoladas de pacientes sintomáticos e axenizadas por Diamond (1968). Utilizamos também a cepa ICB-462, isolada e axenizada diretamente de cistos, de paciente assintomático, isolada e axenizada por um de nós (EFS). Para a prova de HAI, os antígenos foram preparados de acordo com a técnica descrita por KRUPP (1974), e para a IFI seguimos a técnica de GOLDMAN (1966). Os resultados foram considerados positivos quando apresentaram títulos iguais ou superiores a 1:160 pela HAI e 1:64 pela IFI. Estes resultados foram analisados pelo $X^{2}$ e os valores obtidos corrigidos para a continuidade proposta por Yates (AMBROISE-THOMAS, 1976).

\section{RESULTADOS}

Pelas técnicas de HAI e IFI foram detectados, respectivamente, 20,5\% e $26,9 \%$ de indivíduos sintomáticos. Na forma assintomática a positividade foi de $1,3 \%$ para a HAI e 6,6\% para a IFI (tabelas 1 e 2). Todos os soros de indivíduos infectados com outras amebas, diferentes da $E$. histolytica, foram não reativos em ambas as provas. O grupo etário acima de 18 anos representou $30,3 \%$ dos positivos para HAI e $36,4 \%$ para IFI e o grupo etário inferior a 18 anos constituiu $5,8 \%$ e $11,6 \%$, respectivamente. Não foram observadas diferenças significativas entre os sexos feminino e masculino (tabela 3).

Tabela 1. Hemoaglutinação indireta (HAI) em soros de indivíduos infectados com Entamoeba histolytica, segundo a forma clínica.

\begin{tabular}{lccc}
\hline \hline FORMA CLINICA & POSITIVOS $(\geq 1 / 160)$ & NEGATIVOS & TOTAL \\
\hline Sintomática & $16(20,5 \%)$ & $62(79,5 \%)$ & 78 \\
Assintomática & $01(1,3 \%)$ & $75(98,7 \%)$ & 76 \\
\hline \multicolumn{1}{c}{ TOTAL } & $17(11,0 \%)$ & $137(89,0 \%)$ & 154 \\
\hline$X^{2} 1$ gl $=13,16$ & & \\
$\mathrm{p}<0,005$ & & &
\end{tabular}


SILVA, E.F.; GOMES, M.A.; CARVALHO, M.G.Avaliação das técnicas de Hemoaglutinação Indireta e SILVA, E.F.; GOMES, M.A.; CARVÁstico da amebíase intestinal. Rev. Pat. Trop.25 ( 1 ): 5-11, jan/jun. 1996

Tabela 2. Imunofluorescência Indireta (IFI) em soros de indivíduos infectados com Entamoeba histolytica segundo a forma clínica.

\begin{tabular}{cccc}
\hline \hline FORMA CLÍNICA & POSITIVOS $(\geq \mathbf{1} / 64)$ & NEGATIVOS & TOTAL \\
\hline Sintomática & $21(26,9 \%)$ & $57(73,1 \%)$ & 78 \\
Assintomática & $05(6,6 \%)$ & $71(93,1 \%)$ & 75 \\
\hline TOTAL & $26(16,9 \%)$ & $128(83,1 \%)$ & 154 \\
\hline
\end{tabular}

$X^{2} 1 \mathrm{gl}=11,89$

$\mathrm{p}<0,05$

Tabela 3 - Resultados das provas de HAI e IFI segundo idade e sexo dos indivíduos infectados.

\begin{tabular}{lcccccccccc}
\hline \hline & \multicolumn{1}{c}{ HAI } & \multicolumn{4}{c}{ IFI } \\
\hline \hline IDADE & \multicolumn{1}{c}{$\mathbf{N}^{\mathbf{0}}$ DE } & \multicolumn{1}{c}{ POSITIVOS } & \multicolumn{2}{c}{ NEGATIVOS } & \multicolumn{2}{c}{ POSITIVOS } & \multicolumn{2}{c}{ NEGATIVOS } \\
& SOROS & F & M & F & M & F & M & F & M \\
\hline $0-18$ anos & 121 & 4 & 3 & 58 & 55 & 6 & 8 & 53 & 54 \\
$18-66$ anos & 33 & 6 & 4 & 11 & 12 & 6 & 6 & 10 & 11 \\
\hline TOTAL & $\mathbf{1 5 4}$ & $\mathbf{1 7 ( 1 1 , 0 \% )}$ & $\mathbf{1 3 7 ( 8 9 , 0 \% )}$ & $\mathbf{2 6 ( 1 7 , 0 \% )}$ & $\mathbf{1 2 8 ( 8 3 , 0 \% )}$ \\
\hline
\end{tabular}

HAI: $X^{2} 1 \mathrm{gl}=12,55, \mathrm{p}<0,05$

IFI: $X^{2} 1 \mathrm{gl}=9,01, \mathrm{p}<0,05$

$\mathrm{F}=$ feminino $\mathrm{e} M=$ masculino

Não houve diferença significativa $(p<0,05)$ quando os antígenos provenientes das cepas NIH-200 e ICB-462 de $E$. histolyitca, foram comparados com os resultados provenientes da cepa HK-9, empregada como cepa padrão.

\section{DISCUSSÃO}

Utilizando as técnicas de HAI e IFI, observamos um maior número de reaçð̃es positivas nos soros provenientes de indivíduos sintomáticos, quer seja com CND ou $\mathrm{CD}$, que nos assintomáticos. Contudo, analisando as tabelas 1 e 2 , podemos verificar $\mathrm{CD}$, que nos assintomáticos. Contudo, analisando as tas com colite não disentérica não que a maioria dos soros provenientes de sintomáticos com colite nue a forma clínica de foram reativos em nenhuma das de anticorpos tanto para HAI (1/4096 e 1/8192) como CD apresentou títulos elevados de anticorpos tanto para para IFI (1/512 e 1/1024). Esta variação de reatividade pode depender das caracteristicas dos antigenos, da eficiência do hospedeiro em produzir anticorpos, do
SILVA, E.F.; GOMES, M.A.; CARVALHO, M.G.Avaliaçăo das técnicas de Hemoaglutinaçăo Indireta e Imunofluorescência Indireta no diagnóstico da amebíase intestinal. Rev. Pat. Trop.25 ( 1 ): 5-11, jan/jun 1996

tempo de exposição ao parasita, como também pode significar infecção dos hospedeiros por amebas distintas. Considerando esta última hipótese, os indivíduos apresentando a forma clínica de CND poderiam estar infectados com outra espécie de ameba que não a $E$. histolyitca, a $E$. dispar. Esta ameba seria patogênica, contudo não muito virulenta, provocando erosão da parede intestinal sem invadir de forma definitiva a mucosa, como acontece com a $E$. histolytica (CLARK \& DIAMOND, 1994).

Analisando os resultados obtidos com as técnicas de HAI e IFI, observamos que $\mathbf{9 8 , 7 \%}$ e $\mathbf{9 3 , 4} \%$ dos soros dos indivíduos assintomáticos e $79,5 \%$ e $73,1 \%$ dos sintomáticos, não reagiram frente a HAI e IFI, respectivamente. Estes resultados poderiam ser devidos a dois motivos: 1) baixa sensibilidade e especificidade de ambos os testes; 2) existência de cepas, raças, "clones geográficos", ou outra espécie, semelhante a $E$. histolytica, que não invadiria tecidos. $O$ primeiro destes motivos poderia justificar o encontro de reações negativas em indivíduos sintomáticos com CND, e o segundo, os casos assintomáticos e sintomáticos de CND não reativos, uma vez que nestes indivíduos não deve existir invasão tissular.

Neste trabalho empregamos as cepas NIH-200, HK-9 e ICB-462, as duas primeiras isoladas de casos de amebíase sintomática, sendo uma delas a HK-9 usada como cepa padrão e a última, isolada de portador assintomático. Procuramos verificar se a utilização da cepa ICB-462 como antígeno aumentaria a especificidade e sensibilidade no imunodiagnóstico dos indivíduos assintomáticos, como também dos indivíduos apresentando a forma clínica de CND, pouco detectada com a utilização de antígenos obtidos de cepas patogênicas. Não observamos diferença significativa $(\mathrm{p}<$ $0,05)$ quando comparamos os resultados obtidos com os antígenos destas três cepas. Estes resultados sugerem que a forma clínica de CND, muito comum em nosso país, não invade a mucosa intestinal, não sensibilizando o sistema imune do indivíduo infectảdo. No caso dos indivíduos sintomáticos com amebíase ativa (CD ou abscesso hepático), possivelmente ocorre invasão tissular facilitando o contato do antígeno com o sistema imunológico, o que estimula a produção de anticorpos. Portanto, quanto maior a capacidade de invasão do parasita, maior seria a quantidade de antígeno disponível e mais altos os níveis de anticorpos detectados pelas reaçð̃es imunológicas (NEAL et al., 1968). Contudo os indivíduos estudados neste trabalho poderiam não possuir a eficiência nescessária à produção de anticorpos que depende de fatores individuais como, debilidade, má nutrição, deficiência imunológica e mesmo o tamanho do inóculo influenciando no estabelecimento e qualidade da infecção (KRUPP, 1974). Realmente, isto poderia estar ocorrendo uma vez que a maioria destes indivíduos pertencem a um nível sócio-econômico mais baixo. 
SILVA, E.F.; GOMES, M.A.; CARVALHO, M.G.Avaliação das técnicas de Hemoaglutinação Indireta e SILVA, E.F.; GOMES, M.A.; CARVÁtico da amebíase intestinal. Rev. Pat. Trop.25 ( 1 ): 5-11, jan/jun. Imuno
1996

Com relação a idade foi detectada uma maior positividade das reações imunológicas em soros de indivíduos acima de 18 anos, fato este que, provavelmente, se deva principalmente aos hábitos alimentares dos adultos.

Em resumo, embora a maioria dos testes sorológicos empregados no Brasil Eara separar formas assintomáticas das formas sintomáticas de $\mathrm{CD}$, elas não foram capazes no entanto, de diagnosticar a maioria dos sintomáticas de $\mathrm{CD}$, elas náo de CND e assintomática, que são as formas casos de amebíase sintomática de CND e assintomática, que são as formas predom

\section{Agradecimentos}

Agradecemos 0 apoio financeiro dos seguintes órgãos: PRPq-UFMG, FAPEMIG e FINEP

\section{SUMMARY}

Evaluation of Indirect Haemagglutination and Indirect Immunofluorescence on the Diagnosis of Intestinal Amoebiasis.

Tests of indirect haemagglutination (IHA) and indirect immunofluorescence (IIF), were carried out on 154 sera of individuals of both sex with a proven Entamoeba histolytica infection. Amongst these 154 sera, 78 were obtained from patients with clinical symptoms of amoebiasis (disenteric colitis and non disenteric patients with clinical sym 76 sera were from asymptomatic individuals. The antigens colitis) and the remaining 76 sera were from asymptomatic individuals. The antigens were prepared from strains HK- 9 , NIH-200 the IHA and IIF, were analised and axenic culture. The obtained results, both for the IHA and IIF, were analised and related to the patient's clinical presentation, age and sex. A higher positivity was found in the reactions of patients of 18 years and older (IIF:12/21; IHA: 10/23) and with clinical symptoms (IIF: 21/57; IHA: 16/62).

KEYWORDS: Amoebiasis; Diagnosis; Indirect hemagglutination; Indirect immunofluorescence.

\section{REFERÊNCIAS}

01.AMBROISE-THOMAS, P. Immunofluorescence in the diagnosis, therapeutic follow up and seroepidemiological studies of some parasitic diseases. Trans. R. Soc. Trop. Med. Hyg. 70:107 112, 1976.

P. Statistical methods in medical research. London. Blackwell Scientific Publ., 1973, $503 p$
SILVA, E.F.; GOMES, MA ; CARVALHO, M.G.Avaliaço das técnicas de Hemoggtutinąto Indireta Imunofluorescencia Indireta no diagnóstico da amebíase intestinal. Rev. Pat. Trop.25 ( 1 ); 5-11, jan/jun. 1996

03. BRUMPT, E. Étudie sommaire de l'E. dispar n. sp., amiba a kystes quadrinuclées, parasite de l'homme. Bull. Acad. Med. 44:943, 1925.

04.CLARK, C. G. \& DIAMOND, L. S. Pathogenicity, virulence and Entamoeba histolytica. Parasitology Today 10(2):46, 1994

05.CUNHA, A. S.; SILVA, E. F.; ARAÚJO, F. G.; COSTA, C. A.; MELLO, S. M. \& RASO, P. Patogenia da amebíase III - Comportamento de provas sorológicas em pacientes infectados pela Entamoeba histolyitca. Rev. Inst. Med. Trop. São Paulo 19:387-392, 1977.

06.DIAMOND, L. S. Techniques of axenic cultivation of Entamoeba histolytica Schaudinn, 1903 and E. histolytica like amoebae. J. Parasit. 54:1047-1056, 1968.

07.GANDHI, B.M.; IRSHAD, M.; CHARVIA, T. C. \& TANDON, B. Enzyme linked protein-A, an ELISA for detection of amebic antibody. Trans. R. Soc. Trop. Med. Hyg. 81:183, 1987.

08.GOLDMAN, M. Evaluation of a fluorescent antibody for amebiasis ísing two widely differing amoeba strains as antigen. Amer. J. Trop. Med. Hyg. 15:694-700, 1966.

09.GUERRANT, R. L. The global problem of amebiasis: current status, research needs and opportunities for progress. Rev. Infect. Dis. 8:218-223, 1986.

10.HEALY, G. R. Immunological tools in the diagnosis of amoebiasis epidemiology in the United States. Rev. Infect. Dis. 8:239-246, 1986.

11.KESSEL, J. F.; LEWIS, W. P.; MOLINA PASQUEL, C. \& TURNER, J. A. Indirect haemagglutination and complement fixation tests in amebiasis. Am. J. Trop. Med. 14:540-551, 1965.

12.KNOBLOCH, J.; MANNWEILLER, E.; HOELER, W. \& KERN, P. Efficiency of serodiagnosis in amebiasis. Results obtained by for different tests in 1,458 persons with varying exposure. Tropenmed. Parasit. 33:107-110, 1982.

13.KRUPP, L. M. Comparison of counterimmunoelectrophoresis with other serologic tests in diagnosis of amebiasis. Amer. J. Trop. Med. Hyg. 23:27-30, 1974.

14.NEAL, R. A.; ROBINSON, G. L.; LEWIS, W. P. \& KESSEL, J. F. Comparison of clinical observation on patients infected with Entamoeba histolytica with serological titers sera and virulence of other amoebae to rats. Trans. R. Soc. Trop. Med. Hyg. 62:69-75, 1968.

15.SAVANAT, T.; SABCHARDEN, A.; CHARPEMPONG, K.; TANTIVANICH, S. \& KLONGKAMNVANKARN, $\mathrm{K}$. Immunological tests in asymptomatic intestinal amoebiasis. The Southeast Asian J. Trop. Med. Publ. Hlth. 5:359-364, 1974.

16.STAMM, W. P.; ASHLEY, M. J. \& BELL, K. The value of amoebic serology in an area of low endemicity. Trans. R. Soc. Trop. Med. Hyg. 70:49-53, 1976. 